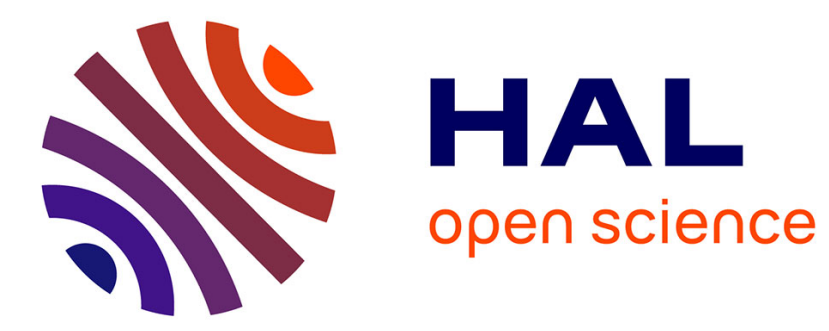

\title{
Une technique de correction du "décalage" d'un échantillon au cours d'expériences sur un diffractomètre à compteur à localisation courbe
}

\author{
F. Bernard, F. Charlot, P. Sarrazin
}

\section{- To cite this version:}

F. Bernard, F. Charlot, P. Sarrazin. Une technique de correction du "décalage" d'un échantillon au cours d'expériences sur un diffractomètre à compteur à localisation courbe. Journal de Physique IV Proceedings, 1996, 06 (C4), pp.C4-103-C4-110. 10.1051/jp4:1996410 . jpa-00254293

\section{HAL Id: jpa-00254293 https://hal.science/jpa-00254293}

Submitted on 1 Jan 1996

HAL is a multi-disciplinary open access archive for the deposit and dissemination of scientific research documents, whether they are published or not. The documents may come from teaching and research institutions in France or abroad, or from public or private research centers.
L'archive ouverte pluridisciplinaire HAL, est destinée au dépôt et à la diffusion de documents scientifiques de niveau recherche, publiés ou non, émanant des établissements d'enseignement et de recherche français ou étrangers, des laboratoires publics ou privés. 


\title{
Une technique de correction du "décalage" d'un échantillon au cours d'expériences sur un diffractomètre à compteur à localisation courbe
}

\author{
F. Bernard*, F. Charlot* et P. Sarrazin*,** \\ * Laboratoire "Réactivité des Solides, URA 23 du CNRS, Faculté des Sciences Mirande, BP. 138, \\ 21004 Dijon cedex, France \\ ** CRITT "Céramiques Fines Techniques", Maubeuge, France
}

\begin{abstract}
Résumé : L'effet d'un décalage de l'échantillon au sein d'un diffractomètre équipé d'un compteur à localisation courbe est analysé. Une technique permettant de corriger l'effet de ces décalages d'échantillon sur le positionnement des raies de diffraction est proposée. Cette technique a été mise en application pour l'exploitation d'expériences diffractométriques en température sur des poudres minérales. Les effets des déplacements d'échantillon, induits par les dilatations thermiques des dispositifs de maintien, ont pu être corrigés permettant des études des évolutions de paramètres cristallins des matériaux malgré l'absence de moyens précis de réglage de la position de l'échantillon.
\end{abstract}

\begin{abstract}
The effect of a sample gap in a diffractometer fitted out with a curve detector is analyzed. A method of correction of the effect of this gap on line positions is proposed. This technique has been used for data analyses of in situ diffraction experiments on mineral powder. The effects on line positions of the sample gap due to the thermal expansion of the sample holder have been corrected allowing cell parameters measurements despite the absence of reliable means of sample adjustement.
\end{abstract}

\section{INTRODUCTION}

La détermination des paramètres cristallins d'un matériau à partir de son diagramme de diffraction passe en général par l'utilisation d'un logiciel d'affinement de paramètres. Ces logiciels calculent les paramètres cristallins qui permettent d'ajuster au mieux l'ensemble des positions expérimentales des raies de diffraction.

Certains logiciels proposent des corrections qui permettent d'intégrer dans l'affinement, en plus des paramètres cristallins, un paramètre représentatif d'une erreur systématique de mesure des positions des raies de diffraction. La correction la plus couramment utilisée est la "correction de zéro" qui corrige une erreur constante sur tout le diagramme de diffraction. On

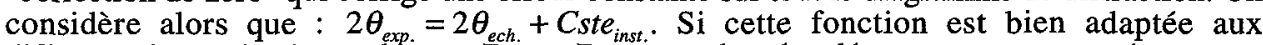
diffractomètres classiques de type Bragg-Brentano dont les détecteurs peuvent présenter un décalage mécanique de zéro, elle n'est pas du tout représentative des erreurs de mesure des positions des raies dans le cas de diffractomètres équipés de compteurs à localisation courbe.

Les diffractomètres à compteurs courbes montrent une forte sensibilité aux décalages que peut présenter l'échantillon par rapport à sa position de référence (position de l'échantillon étalon). Ainsi, tout décalage de l'échantillon au cours d'une série de mesure impose soit, d'effectuer un réglage précis de la position du porte échantillon avant chaque enregistrement, soit, de corriger les positions angulaires mesurées de l'effet du décalage.

Certains dispositifs diffractométriques sont équipés d'enceintes d'étude in situ qui ne permettent pas un réglage précis de la position de l'échantillon avant chaque enregistrement. Ainsi, des études fines des évolutions de paramètres de maille imposent de corriger les positions angulaires des raies de diffraction des effets du décalage éventuel de l'échantillon.

Une technique simple, reposant sur l'analyse des variations des positions angulaires des raies de diffraction d'un échantillon au cours d'un traitement, a été mise au point pour permettre de calculer les variations relatives de paramètres de maille. Cette technique ne passe pas par un calcul des paramètres de maille associés à chacun des enregistrements mais par un ajustement de 
la courbe expérimentale de décalage angulaire des raies de diffraction $\Delta 2 \theta=f(2 \theta)$ par une fonction qui intègre le décalage angulaire associé à un décalage de l'échantillon et le décalage angulaire associé à une variation relative de paramètre cristallin.

\section{PRINCIPE DE LA TECHNIQUE DE CORRECTION DE L'EFFET D'UN DÉCALAGE D'ÉCHANTILLON.}

\section{2-1 Effet d'un décalage d'échantillon sur les positions des raies de diffraction.}

La figure 1 présente le schéma d'un dispositif diffractométrique équipé d'un compteur à localisation courbe. Un faisceau incident irradie un échantillon sous un angle d'incidence $\alpha$, un faisceau diffracté émerge de l'échantillon avec un angle $2 \theta_{0}$ par rapport à la direction du faisceau incident. Lorsque le diffractomètre est parfaitement réglé, l'angle mesuré par le compteur est $2 \theta_{0}$.

Le compteur à localisation courbe nécessite un étalonnage à l'aide d'un échantillon pulvérulent de caractéristiques cristallines bien connues. Du fait de cet étalonnage, la position de référence de l'échantillon est la position de l'échantillon étalon lors de l'enregistrement du diffractogramme d'étalonnage. Pour les calculs, la position de l'échantillon étalon est assimilée au centre goniométrique, condition que I'on cherche dans la pratique à respecter. Un léger défaut de positionnement de l'échantillon étalon ne changerait toutefois que d'une manière négligeable les résultats des calculs.

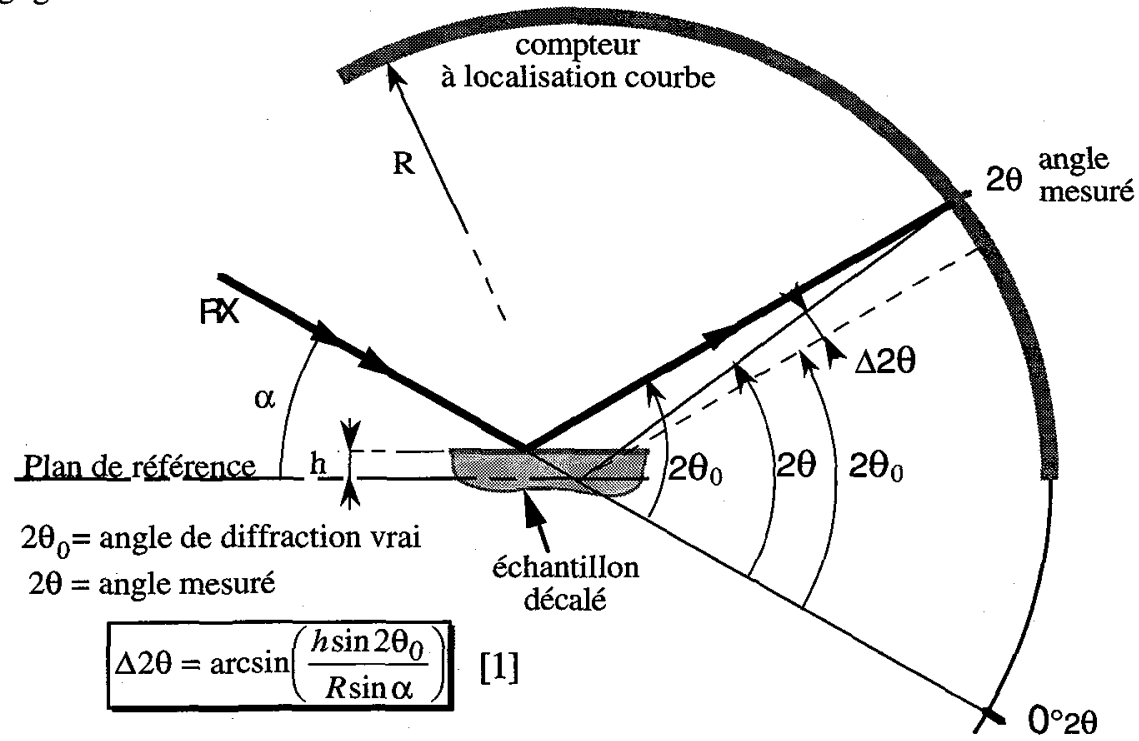

Figure 1 : Schématisation de la diffraction par un échantillon plan et de la mesure de la position angulaire de la raie de diffraction dans le cas où l'échantillon présente un défaut de positionnement $h$; la position angulaire de la raie ne correspond plus à l'angle de diffraction.

Si l'échantillon est décalé d'une valeur $h$ perpendiculairement au plan de référence, un faisceau diffracté émerge toujours de l'échantillon sous un angle de diffraction $2 \theta_{0}$. Toutefois, ce faisceau est décalé par rapport à celui qui apparaît dans le cas d'un réglage parfait. La position angulaire $2 \theta$ mesurée par le compteur ne correspond donc pas à l'angle de diffraction vrai caractéristique du matériau.

Le décalage angulaire $\Delta 2 \theta=2 \theta-2 \theta_{0}$ engendré par un décalage $h$ de l'échantillon est donné par la relation [1].

remarque : En pratique, la relation [1] est bien approchée par $\Delta 2 \theta=\frac{h}{R} \times \frac{\sin 2 \theta_{0}}{\sin \alpha}$

Le décalage angulaire associé à un décalage de l'échantillon varie selon une fonction sinus de l'angle de diffraction $2 \theta_{0}$. On note par ailleurs que l'effet du décalage de l'échantillon est d'autant plus important que l'angle d'incidence est faible. Ceci vérifie la nécessité d'une très 
bonne maîtrise de la position de l'échantillon lors d'études diffractométriques en incidence rasante ( $\alpha$ très faible).

Vérification expérimentale de la relation [1]

La loi de variation des positions angulaires des raies de diffraction sous l'effet d'un décalage $h$ de l'échantillon perpendiculairement au plan de référence a été vérifiée expérimentalement.

Un diffractomètre équipé d'un compteur à localisation courbe INEL CPS 120 et disposant d'une vis micrométrique de réglage fin de la position du porte échantillon a été utilisé. La figure 2 présente les décalages angulaires des raies d'un échantillon de $\mathrm{ZnO}$ en fonction de $2 \theta$ pour différents décalages imposés à l'échantillon.

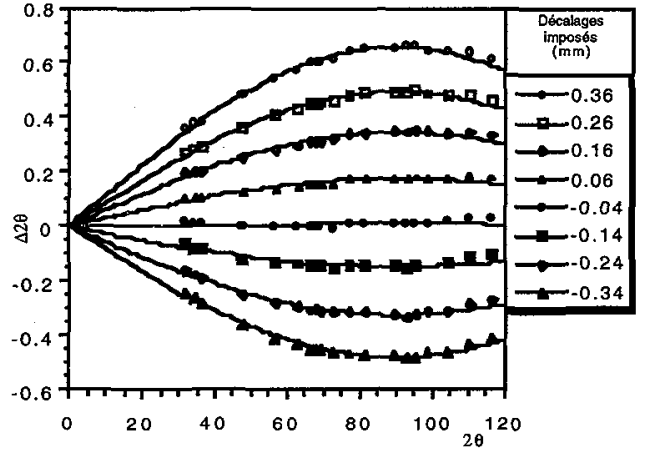

Figure 2 : Décalages angulaires des raies de diffraction en fonction de $2 \theta$ pour différents décalages verticaux imposés à un échantillon de $\mathrm{ZnO}\left(\alpha=8^{\circ}\right)$; ajustements des décalages angulaires par la fonction [1].

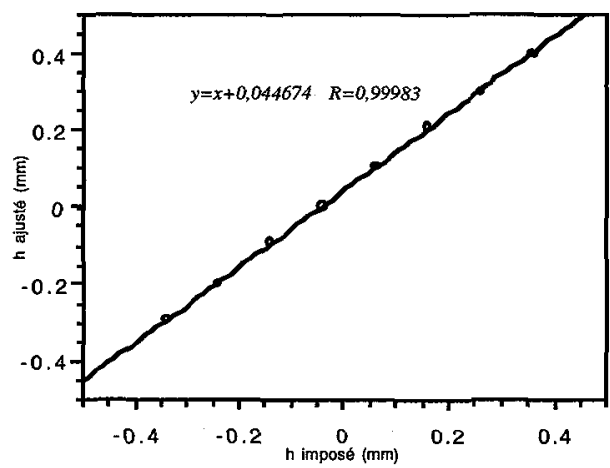

Figure 3 : Décalages d'échantillon ajustés en fonction des décalages imposés à l'échantillon de $\mathrm{ZnO}$.

Les valeurs expérimentales ont été ajustées avec la fonction [1] avec le décalage $h$ pour paramètre d'ajustement. Les ajustements, portés sur la figure 2 , suivent bien les évolutions expérimentales observées.

Les décalages d'échantillon déterminés par les ajustements sont présentés sur la figure 3 en fonction des décalages imposés à l'échantillon. On remarque la très bonne corrélation entre les valeurs imposées et les valeurs calculées, hormis un décalage systématique constant. Ce décalage constant s'est révélé être lié au fait que l'échantillon de référence a été enregistré dans un porte-échantillon différent de celui utilisé pour l'enregistrement des échantillons subissant le décalage. Bien que de géométries identiques, les deux porte-échantillons présentaient une différence de hauteur d'environ $4 / 100 \mathrm{~mm}$ que l'on retrouve évidemment dans le décalage vertical global. Cette observation illustre la bonne précision de la technique de détermination des décalages d'échantillons.

\subsection{Effet d'une variation de paramètre de maille sur les positions des raies de diffraction.}

Dans le cas d'un échantillon polycristallin présentant un réseau cristallin cubique, les variations relatives des distances interréticulaires $\Delta \mathrm{d} / \mathrm{d}_{0}$ sont égales à la variation relative du paramètre de maille $\Delta \mathrm{a} / \mathrm{a}_{0}$.

Les variations de positions des raies de diffraction à l'issu d'une variation relative de paramètre cristallin $\Delta \mathrm{a} / \mathrm{a}_{0}$ peuvent être calculées grâce à la relation de Bragg. On obtient :

$$
\Delta 2 \theta=2 \arcsin \left(\frac{\sin \theta_{0}}{1+\frac{\Delta a}{a_{0}}}\right)-2 \theta_{0}
$$

où $\theta_{0}$ est l'angle de Bragg d'une raie de diffraction du matériau dans son état initial et $\theta$ l'angle de Bragg de la même raie dans l'état final. 


\subsection{Effets conjoints d'un décalage de l'échantillon et d'une variation de paramètre de maille sur les positions des raies de diffraction.}

Dans le cas où un décalage d'échantillon et une variation de paramètre cristallin se produisent simultanément, les raies de diffraction vont être décalées sous les actions conjointes de chacune de ces causes.

La figure 4 montre que les deux causes de décalages angulaires des raies de diffraction sont caractérisées par des effets qui évoluent de manière très différentes avec 20. Dans le cas où les deux effets coexistent, il est donc possible de distinguer ces deux contributions.
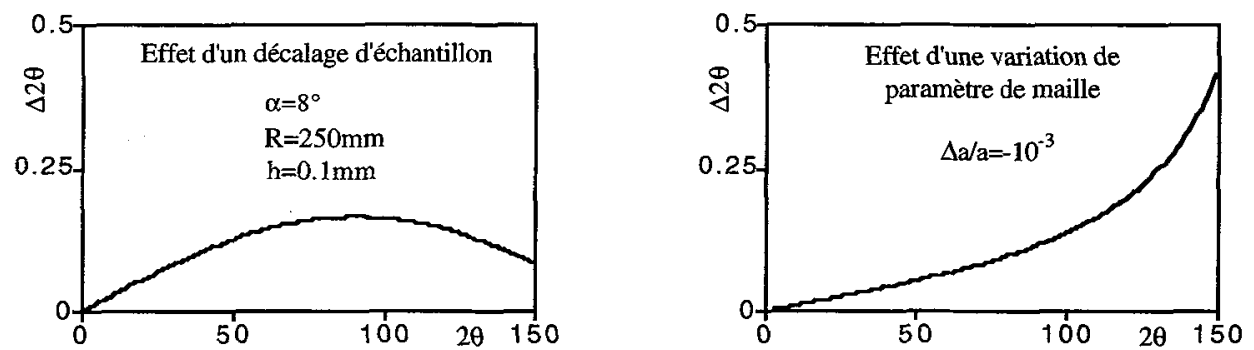

Figure 4 : Évolution avec $2 \theta$ des décalages angulaires des raies de diffraction engendrés par un décalage de l'échantillon seul et par une variation de paramètre de maille seule.

Le décalage angulaire global des raies de diffraction s'exprime par la relation :

$$
\Delta 2 \theta=2 \arcsin \left(\frac{\sin \theta_{0}}{1+\Delta a / a_{0}}\right)+\arcsin \left(\frac{h \sin \left(2 \arcsin \left(\frac{\sin \theta_{0}}{1+\Delta a / a_{0}}\right)\right)}{R \sin \alpha}\right)-2 \theta_{0}
$$

avec : $\theta_{0}=$ angle de Bragg d'une raie du diffractogramme initial, $\theta=$ angle de Bragg de la même raie dans le diffractogramme final, $\mathbf{R}=$ rayon du goniomètre $(250 \mathrm{~mm}$ pour INEL CPS $120), \mathbf{h}=$ décalage vertical de l'échantillon, $\alpha=$ angle d'incidence du faisceau incident, $\Delta \mathbf{a} / \mathbf{a}_{0}$ $=$ variation relative du paramètre de maille.

Lorsqu'une variation de paramètre cristallin d'un matériau à réseau cubique et un décalage de l'échantillon se produisent entre deux enregistrements diffractométriques, la relation [2] est utilisée pour ajuster les décalages de positions angulaires des raies de diffraction observés. L'ajustement permet de calculer la variation relative de paramètre de maille ainsi que le décalage de l'échantillon.

\subsection{Correction pour un affinement "classique" de paramètres de maille.}

Effectuer un affinement de paramètres consiste à calculer le ou les paramètres de maille qui conduisent aux angles de diffraction qui ajustent au mieux les positions expérimentales des raies de diffraction. Dans le cas où une erreur systématique se produit dans la mesure des positions angulaires des raies, il convient de corriger les positions expérimentales par une fonction de correction qui rend compte de l'effet de l'erreur systématique.

La fonction de correction à adopter lorsque l'on utilise un diffractomètre équipé d'un compteur à localisation courbe et qu'il existe un risque de décalage de l'échantillon est alors :

$$
\theta_{\text {corrigé }}=\theta_{\text {exp. }}-\frac{h}{2 R} \frac{\sin 2 \theta}{\sin \alpha}
$$
$\left(\frac{h}{2 R} \frac{\sin 2 \theta}{\sin \alpha}\right)$ est le terme correctif qui doit être ajusté lors de l'affinement de paramètres de
maille avec $h$ pour paramètre d'ajustement. 


\section{EXEMPLES D'APPLICATIONS DE LA TECHNIQUE.}

Nous avons utilisé la technique d'analyse décrite précédemment à chaque fois qu'un décalage de l'échantillon était susceptible de se produire au cours d'une série d'enregistrements diffractométriques d'un même échantillon. La méthode a en particulier montré son utilité pour les études d'évolutions de paramètres cristallins de poudres en fonction de la température, les dilatations des dispositifs de maintien des échantillons au sein des fours ou cryostats étant alors à l'origine de décalages de l'échantillon.

Deux exemples pratiques relatifs à des études menées sur le titanate de strontium $\mathrm{SrTiO}_{3}$ illustrent l'utilisation de la technique de correction.

\subsection{Expériences effectuées sur un diffractomètre équipé d'une chambre haute température}

Un diffractomètre équipé d'un compteur à localisation courbe INEL CPS 120 et d'une chambre haute température à atmosphère contrôlée a été utilisé pour mesurer l'évolution avec la température du paramètre cristallin d'une poudre de $\mathrm{SrTiO}_{3}$ (figure 5).

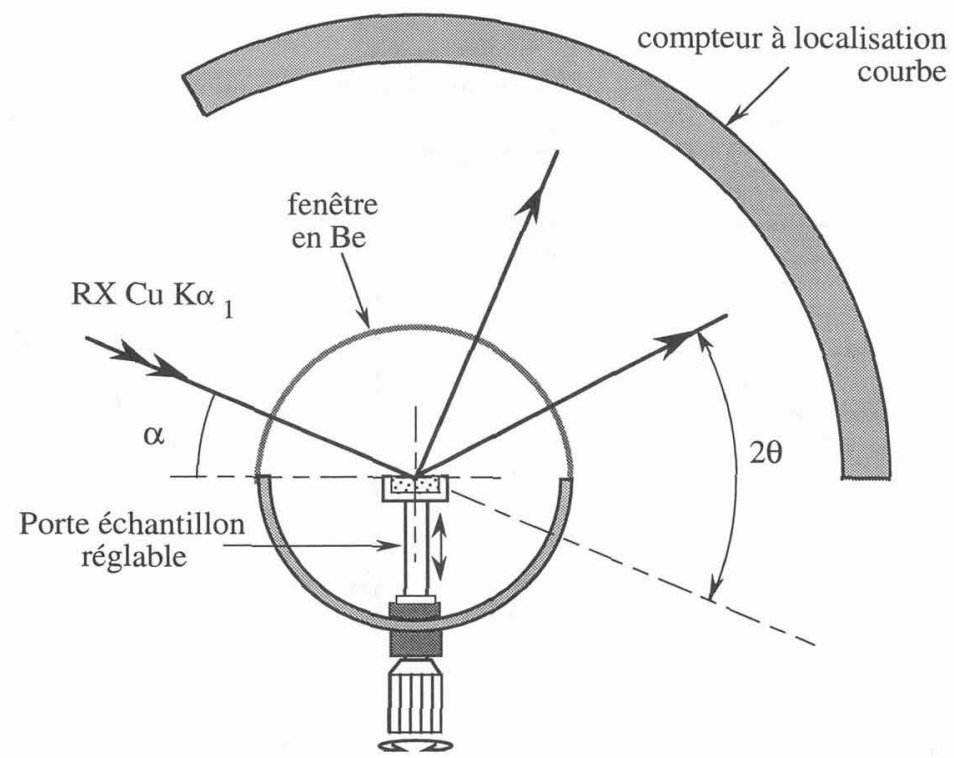

Figure 5: Schéma du dispositif diffractométrique équipé d'un compteur à localisation courbe et d'une chambre haute température à atmosphère contrôlée.

L'élévation de température de la chambre a pour conséquence de dilater le porte échantillon, ce qui entraîne un décalage de l'échantillon. La mauvaise précision du dispositif de réglage et l'absence de moyen fiable de contrôle de la position de l'échantillon lorsque l'enceinte est. fermée ont conduit à utiliser la méthode de correction décrite précédemment.

Les positions angulaires des raies de diffraction de l'échantillon ont été enregistrées à différentes températures. Les différences de position des raies de diffraction $\Delta 2 \theta$ entre chaque enregistrement en température et l'enregistrement initial effectué à la température ambiante dans des conditions de réglage optimales ont été calculées. Chaque courbe $\Delta 2 \theta=f(2 \theta)$ a été ajustée par la fonction [2] à l'aide du logiciel Kaleidagraph afin de calculer la différence relative de paramètres cristallin $\Delta \mathrm{a} / \mathrm{a}_{0}$ (par rapport au paramètre du matériau à la température ambiante) et le décalage $\mathrm{h}$ associé à la dilatation du porte échantillon. La figure 6 montre une des courbes expérimentales $\Delta 2 \theta=\mathrm{f}(2 \theta)$ et son ajustement par la fonction [2]. L'allure de la courbe montre la 
coexistence d'une modification de paramètre de maille (dilatation de l'échantillon) et d'un décalage de l'échantillon (dilatation du porte échantillon).

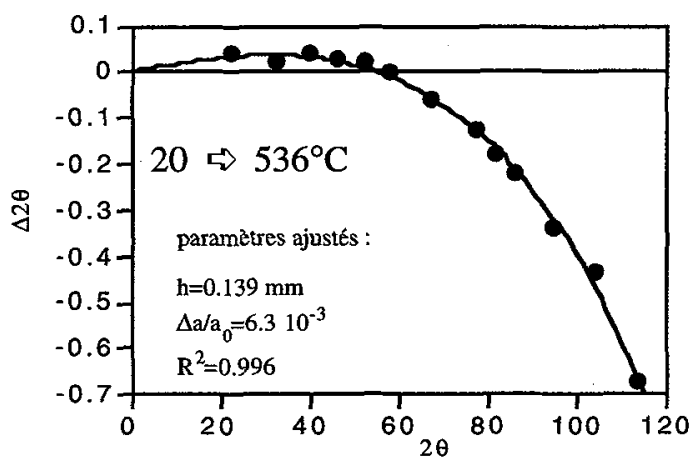

Figure 6 : Évolution en fonction de $2 \theta$ des décalages des raies de diffraction mesurés entre l'ambiante et $536^{\circ} \mathrm{C}$; ajustement des décalages angulaires par la fonction [2].

Les différents ajustements ont permis d'établir les graphes des figures 7 et 8 montrant respectivement l'évolution relative du paramètre cristallin de l'échantillon et l'évolution du décalage de l'échantillon en fonction de la température.

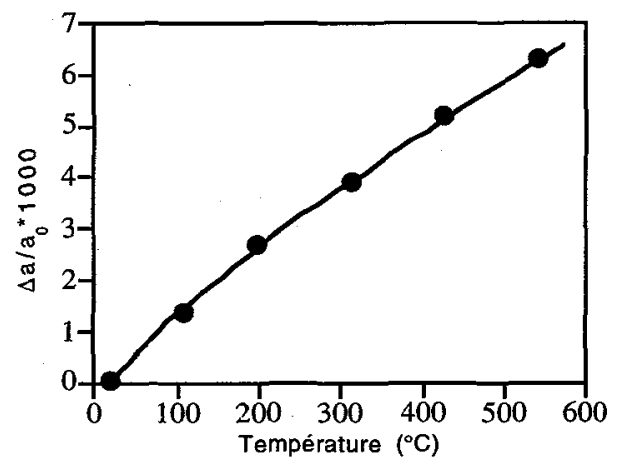

Figure 7 : Évolution avec la température de la variation relative de paramètre de maille mesurée par ajustement des courbes $\triangle 2 \theta=f(2 \theta)$.

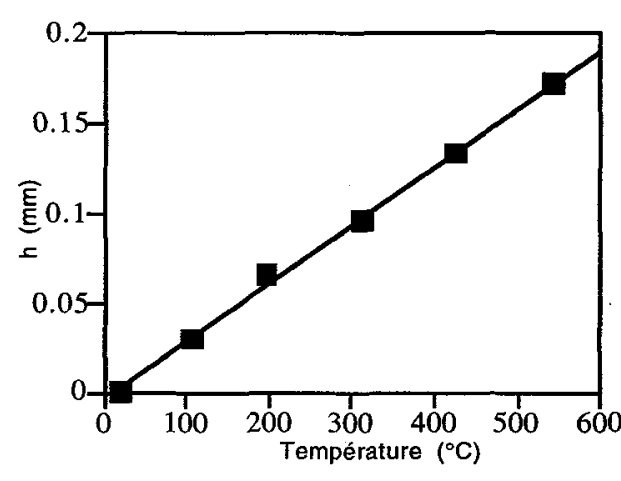

Figure 8 : Évolution avec la température du décalage d'échantillon mesuré par ajustement des courbes $\Delta 2 \theta=f(2 \theta)$.

Le décalage de l'échantillon, linéaire avec la température, est cohérent avec une dilatation linéaire du porte-échantillon. L'évolution relative de paramètre cristallin de $\mathrm{SrTiO}_{3}$ montre une diminution de pente lorsque la température est augmentée. Cette évolution, contraire à la tendance générale des matériaux, a été vérifiée par mesure dilatométrique sur barreau céramique de ce matériau.

\subsection{Expériences effectuées sur un diffractomètre équipé d'une chambre basse température}

Des mesures diffractométriques d'une poudre de $\mathrm{SrTiO}_{3}$ ont été effectuées dans une enceinte cryogénique au cours d'une montée en température par paliers de $-160^{\circ} \mathrm{C} a ̀+90^{\circ} \mathrm{C}$. Dans tout ce domaine de température, la structure de $\mathrm{SrTiO}_{3}$ reste cubique.

Le porte-échantillon dans l'enceinte cryogénique est conçu d'une manière différente de celui du four haute température. En effet, l'axe du porte échantillon dans la chambre haute température est perpendiculaire à l'axe goniométrique de la platine, alors que l'axe du porte- 
échantillon dans l'enceinte cryogénique est colinéaire à l'axe du goniomètre. La direction principale de dilatation est alors transversale par rapport au plan d'incidence des rayons X. Son effet sur le décalage de l'échantillon doit être minimisé.

Le paramètre de maille de l'échantillon a été calculé pour chaque enregistrement par un affinement classique grâce au logiciel Celref. L'évolution du paramètre ainsi calculé en fonction de la température est présentée sur la figure 9.

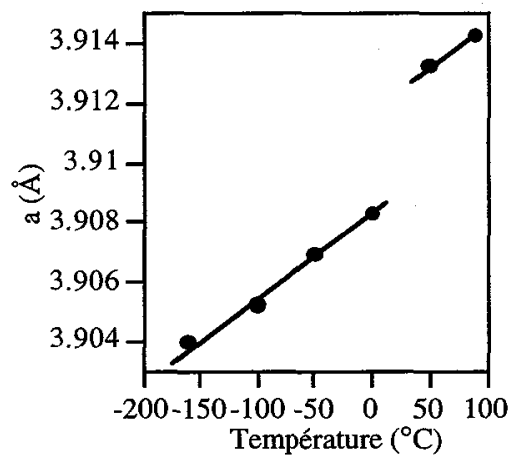

Figure 9 : Variation en fonction de la température de paramètre de maille de $\mathrm{SrTiO}_{3}$ calculé par un affinement classique.

Une discontinuité de la courbe apparaît dans la zone $0^{\circ} \mathrm{C} / 50^{\circ} \mathrm{C}$ alors qu'aucune transition de phase de $\mathrm{SrTiO}_{3}$ n'existe dans ce domaine de température. La discontinuité observée découle manifestement d'un problème expérimental mais l'analyse effectuée ne permet pas d'en déterminer l'origine.

Les résultats de ces expériences ont fait l'objet d'une deuxième analyse selon la technique décrite dans les paragraphes précédents. L'enregistrement à la température de $0^{\circ} \mathrm{C}$ est la référence des positions des raies. Pour chaque température, la fonction de correction [2] est ajustée aux points expérimentaux à l'aide du logiciel Kaleidagraph, elle permet ainsi de déterminer la variation relative du paramètre de maille $\Delta a / a_{0}$ et un éventuel décalage $h$ du porteéchantillon. La figure 10 montre l'évolution du décalage $h$ en fonction de la température, la figure 11 présente l'évolution de la variation du paramètre de maille en $\Delta a / a_{0}$ en fonction de la température.

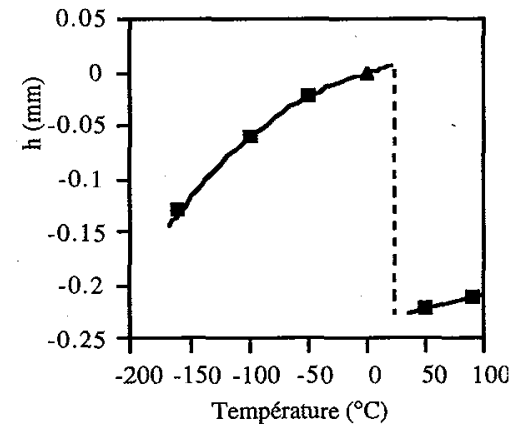

Figure 10 : Décalage $\mathrm{h}$ de l'échantillon en fonction de la température.

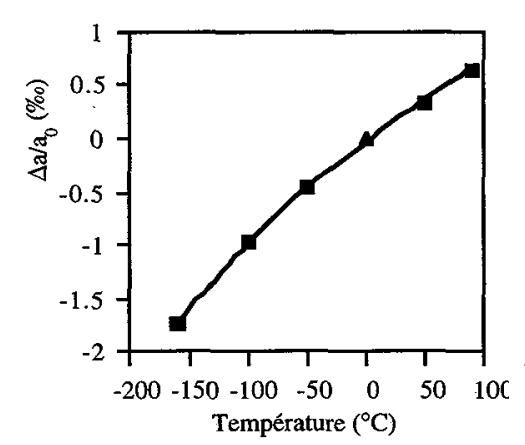

Figure 11 : Variation de paramètre de maille $\Delta \mathrm{a} / \mathrm{a}_{0}$ en fonction de la température.

Les valeurs de $\Delta \mathrm{a}_{\mathrm{a}} \mathrm{a}_{0}$ calculées ont permis de déterminer le paramètre de maille pour chaque enregistrement. La figure 12 présente l'évolution en fonction de la température du paramètre de maille ainsi calculé. Les points relatifs aux affinements "classiques" de paramètre de maille (Celref sans correction de zéro) sont reportés sur le graphe pour permettre une comparaison des résultats des deux méthodes. 


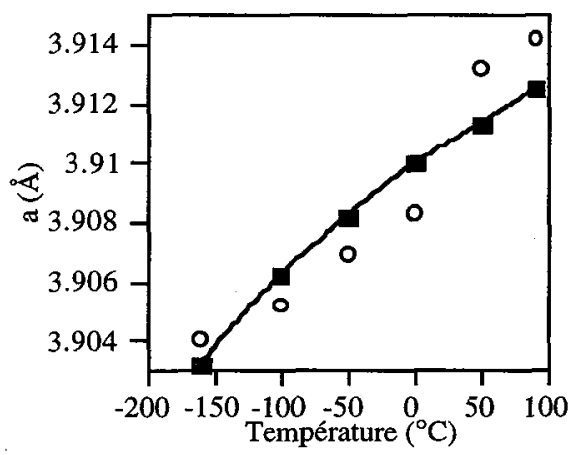

Figure 12: Évolution du paramètre de maille avec la température :

affiné par la technique de correction,

$O$ affiné par la technique classique.

L'évolution du paramètre de maille déterminée par la méthode de correction, monotone avec la température, est en accord avec le comportement attendu du matériau. La figure 10 met par ailleurs en évidence l'existence d'un décalage de l'échantillon dont l'évolution montre une discontinuité entre $0^{\circ} \mathrm{C}$ et $50^{\circ} \mathrm{C}$. Cette observation correspond à un changement brutal de la position de l'échantillon au cours de l'expérience.

La méthode de correction a ici permis de mettre en évidence un "accident expérimental" et d'en corriger l'effet pour obtenir l'évolution effective du paramètre de maille du matériau étudié.

\section{CONCLUSION}

La validité de la technique d'exploitation présentée a été vérifiée par différentes études expérimentales sur différents diffractomètres équipés de compteurs à localisation courbe. Grâce à des calculs simples, cette méthode permet une détermination fiable des évolutions des paramètres cristallins de matériaux à réseau cubique en s'affranchissant des problèmes causés par d'éventuels décalages de l'échantillon.

Cette technique a en particulier montré son efficacité pour les expériences diffractométriques in situ qui présentent des risques accrus de décalage de l'échantillon et des difficultés de réglage de sa position.

L'utilisation d'un logiciel d'affinement de paramètres permettant d'intégrer la fonction de correction décrite dans l'équation [3] devrait permettre d'obtenir les mêmes performances sans pour autant se limiter aux matériaux à réseau cristallin cubique. 